\title{
Current status of technique for Billroth-I anastomosis in totally laparoscopic distal gastrectomy for gastric cancer
}

\author{
Shun Zhang ${ }^{1,2}$, Tetsu Fukunaga ${ }^{1}$ \\ 1Department of Gastroenterology and Minimally Invasive Surgery, Juntendo University Hospital, Tokyo 113-8421, Japan. \\ ${ }^{2}$ Department of Gastroenterology Surgery, Shanghai East Hospital, Shanghai 200120, China.
}

Correspondence to: Dr. Tetsu Fukunaga, Department of Gastroenterology and Minimally Invasive Surgery, Juntendo University Hospital, 3-1-3 Hongo, Bunkyo-ku, Tokyo, Japan. E-mail: t2fukunaga@juntendo.cac.jp

How to cite this article: Zhang S, Fukunaga T. Current status of technique for Billroth-I anastomosis in totally laparoscopic distal gastrectomy for gastric cancer. Mini-invasive Surg 2019;3:2. http://dx.doi.org/10.20517/2574-1225.2018.64

Received: 2 Oct 2018 First Decision: 23 Oct 2018 Revised: 19 Dec 2018 Accepted: 24 Dec 2018 Published: 14 Jan 2019

Science Editor: Tetsu Fukunaga Copy Editor: Cui Yu Production Editor: Huan-Liang Wu

\begin{abstract}
Several reconstruction techniques are possible after totally laparoscopic distal radical gastrectomy. An optimal technique of digestive tract reconstruction after distal gastrectomy has not yet been established. The ideal reconstruction should be not only for doctors but also for patients. Alimentary intake, satisfactory nutritional status and easy performing should be all considered. The aim of the study was to describe the different Billroth-I reconstruction techniques that can be proposed after totally laparoscopic distal radical gastrectomy.
\end{abstract}

Keywords: Billroth-I anastomosis, totally laparoscopic distal gastrectomy, gastric cancer

\section{INTRODUCTION}

In 1994, Kitano firstly reported the technique for laparoscopy assisted Billroth-I (B-I ${ }^{[1]}$. Since then, the use of laparoscopic treatments for gastric cancer is increasing due to the advantages of improving patients' quality of life. The new technologies and improved techniques have allowed laparoscopy gastrectomy to expand its indications and also to use this treatment for more complex cases. Japan Society of Endoscopic Surgery (JSES) conducted national survey every 2 years and indicated the percentage of laparoscopic procedures for gastric cancer was increasing. According to the 12th JSES survey, laparoscopic distal gastrectomy (LDG) was the most commonly performed type of laparoscopic gastrectomy ${ }^{[2]}$.

In initial series for LDG, the majority of anastomoses were performed by laparoscopy assisted procedures.

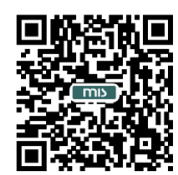


The assisted procedures needed a mini-laparotomy incision of 60-70 $\mathrm{mm}$ in length made on the epigastrium $^{[3]}$. But this procedure was not always easy to do, especially on patients with a small remnant stomach or obese patients with thick abdominal walls ${ }^{[4]}$. Anastomosis in such restricted space was usually difficult. With the accumulation of laparoscopic surgery experience and the development of laparoscopic devices, the gastrointestinal reconstruction now can be completed laparoscopically. Furthermore, unnecessary manipulations and the incision made on the epigastrium can be avoided.

The ideal reconstruction should be not only for doctors but also for patients. Alimentary intake, satisfactory nutritional status and easy performing should be all considered ${ }^{[5]}$. The B-I anastomosis is preferred by many doctors. It is said that the B-I anastomosis is simple and can provide a physiological route for food digestion and absorb without the need for an intestinal bypass or blind loop. Until now, various intracorporeal B-I anastomosis techniques were reported. In this article, we will review theses reconstruction methods.

\section{HAND-SEWN ANASTOMOSIS IN INTRACORPOREAL B-I RECONSTRUCTION}

After the accumulation of operative experience, some experienced surgeons had also presented intracorporeal hand-sewn techniques.

Takiguchi et al. ${ }^{[6]}$ firstly reported B-I intracorporeal hand-sewn anastomosis in 2003. In his study, the Albert-Lembert method was used for the laparoscopic hand-sewing procedure and the anastomosis time was $90 \mathrm{~min}$. Due to the complexity of the procedure and large amount of time required for anastomosis, it seemed that the hand-sewn anastomosis was not widely performed.

After almost 10 years, Matsuo et al. ${ }^{[7]}$ reported another study about hand-sewn B-I anastomosis. They performed hand-sewn gastroduodenal anastomosis in 18 cases. The mean time of B-I anastomosis was 64.6 min. Matsuo et al. ${ }^{[7]}$ described that 3-0 absorbing thread was placed in the lesser curvature as a supporting thread. A seromyotomy of the stomach was performed at the posterior wall. Both the remmant stomach and the duodenum's seromuscular layer were discontinuously sutured by extracorporeal knottyping method. The lumen was opened with the stomach and the duodenum in a fixed status. The thread of the anchor suture was lifted upward to the abdominal wall. After that all layers of the stomach and the duodenum at the posterior wall were continuously sutured. The authors believed that hand-sewn anastomosis had some advantages. Hand-sewn sutures were not affected by the degree of freedom of the duodenum. Because staplers were not used, the anastomosis area was soft and highly flexible. The handsewn anastomosis was economical due to that less staplers were used.

\section{CIRCULAR STAPLER USED IN INTRACORPOREAL B-I RECONSTRUCTION}

In the open surgery, circular stapler is well applied as a standardized reconstruction method of gastroduodenostomy. However, when it was attempted laparoscopically, the situation was often the opposite.

Uyama et al. ${ }^{[8]}$ firstly described intracorporeal B-I reconstruction using a circular stapling device and introduced one case in 1995. The method was defined by the same anatomic parameters as for the open B-I. After that, Moriya et al. ${ }^{[9]}$ and Mayers and Orebaugh ${ }^{[10]}$ also reported B-I gastroduodenostomy with a circular stapler device. Both techniques were complicated and difficult to operate, and especially at the left subcostal area where an extended incision was needed. The extra incision spoiled the merit of minimally invasive surgery.

There are 2 major difficulties when circular stapler is applied in laparoscopic gastroduodenostomy: the first is the lack of a safe and fast intracorporeal purse-string suture technique and the second is the difficulty 
in manipulating the stapler and the stomach in a narrow abdominal cavity. In order to enable the anvil placement into the dudenum, many strategies were applied, such as a triple stapling procedure ${ }^{[8]}$ and the use of the natural pyloric ring with endo-looping of the duodenum ${ }^{[9]}$. Some techniques usually used in esophagoenteral anastomosis were also reported, such as using specially modified laparoscopic pursestring instrument ${ }^{[11]}$ and opening the lumen and applying manual purse-string suture ${ }^{[12]}$. However, there are still many difficulties to be overcome.

Kim et al. ${ }^{[13]}$ reported a method which seemed to be quick and economical. The atraumatic clamps were used to prevent slippage of the duodenum which was cut with ultrasonic shear instead of linear stapler. After that, a seromuscular suture was done around the duodenal outer layer along the clamp. Omori el al. ${ }^{[14]}$ reported a method like reverse puncture technique used in total gastrectomy. The anvil secured with vicryl suture was inserted into the duodenum through semicircumferential duodenotomy. The needle was advanced to the anterior duodenal wall and then the duodenum was staple-transected. Finally, the center rod penetrated the duodenal wall. In this method the need for purse-string suture placement was totally eliminated.

Although the skill inserting anvil head in the duodenal stump can be improved, laparoscopically inserting the circular stapler into the remnant stomach was not always easy. After removing two-thirds to threequarters of the stomach, the small remnant stomach was usually so small that it was difficult to insert the stapler, even from the epigastric region. Sometime it was very difficult to form a straight line among the duodenum, remnant stomach and the circular stapler from the umbilical wound. Omori el al ${ }^{[15]}$ described a novel method to insert the circular stapler to connect the anvil head. Firstly, the anvil head was passed through the posterior gastric wall with laparoscopic endloop, which can make the duodenum and remnant stomach form a straight line. Secondly anterior gastric suture was used to exteriorize the anvil shaft partly from the gastrotomy. And then the anvil shaft was advanced into the remnant stomach to make the anvil and the stapler join tighter.

\section{LINEAR STAPLER INTRACORPOREAL B-I RECONSTRUCTION}

\section{Delta-shaped anastomosis and modified delta-shaped anastomosis}

With the development of laparoscopic instruments and the continuous accumulation of surgical experience in recent years, linear stapler intracorporeal gastrointestinal anastomosis techniques have been developed.

Kanaya et al. ${ }^{[16]}$ firstly reported a anastomosis method which used only laparoscopic linear staplers in the hope of overcoming the drawbacks of extracorporeal reconstruction. The method named delta-shaped anastomosis (DA) was a modified intracorporeal B-I reconstruction which was soon promoted. The emergence of the DA method made intracorporeal gastroduodenostomy possible, which greatly promoted the development of totally laparoscopic distal gastrectomy (TLDG). Utilization DA method allows gastroduodenal anastomosis with a diameter of at least $30 \mathrm{~mm}$ while avoiding stricture. Kanaya et al. ${ }^{[17]}$ analyzed the result of initial 100 procedures and showed that the mean time of the anastomosis was 13 min and the rate of anastomosis related complications was rare in 2011.

But some surgeons worry about the blood supply affected during cutting, which would result in leakages ranging from $0.42 \%$ to $8.5 \%{ }^{[17-20]}$ and anatomical distortion which exist in twisting around the anastomosis $^{[21]}$. In order to overcome the twisting around the anastomosis, some modified delta-shaped techniques were studied.

Huang et al. ${ }^{[22]}$ reported modified DA in 2014. This was different from the conventional DA in closing the common stab incision of stomach and duodenum. In order to avoid the poor blood supply of the duodenum, the duodenal cutting was totally resected. The appearance of the anastomoses was changed 
from two intersections to only one as an inverted T-shape, which could decrease the anastomotic weak point. They reported comparable postoperative outcomes and showed that modified DA was technically safe and feasible ${ }^{[23]}$ in another study.

After DA, many surgeons develop many other anastomosis methods based on linear stapler.

\section{Triangulating stapling technique}

Tanimura et al. ${ }^{[24]}$ described the triangulating stapling technique based on a linear stapling device in 2008. The mean anastomotic time was $35 \mathrm{~min}$. In this method, the duodenum can be transected in any direction, and by forming a triangle, the anastomosis lumen is made wide with no ischemic areas. Both stumps of duodenum and remnant stomach were opened fitting their caliber, the gastroduodenostomy linear stapler in the posterior wall and 2 everted sutures in the anterior wall with linear staplers. Before each direction anastomosis, both duodenum and remnant stomach were elevated ventrally with 3 stay sutures. But there are still some problems about this method. There were some differences between the stomach and duodenum in terms of lumen size, wall thickness, and wall extensibility. The first introverted anastomosis, which forms the base of the triangle, was cumbersome once all of the staple lines on the stomach and duodenum had been cut off.

\section{Book-binding technique}

Ikeda el al ${ }^{[25]}$ described the book-binding technique using linear stapler in 2012. The mean anastomotic time was $34 \mathrm{~min}$. In their method, the duodenum was transected form the greater curvature side to the lesser curvature side. Small openings are made in the remnant stomach and duodenal stumps just wide enough to insert one of the jaws of the linear stapler. After the first stapling, there were three staple lines including those from the transection of the stomach and duodenum, which ran in parallel to the anterior wall. To prevent the formation of ischemic areas, a large opening was created on the anterior wall by transecting the entire duodenal stump and one-third of the gastric stump together with the anterior wall of the first anastomosis line. The anterior hole was then fired by linear stapler twice to close the large opening. Because a large opening was created on the anterior wall by transecting tissue and anastomosis line, maybe some tension was generated after the anterior hole was closed by the linear stapler. Further studies need to be done.

\section{Linear-shaped gastroduodenostomy}

Byun et al. ${ }^{[26]}$ developed a linear-shaper gastroduodenostomy method by which the appearance of anastomosis was completed inverted T-shaped in 2009. Duodenum was transected from the greater curvature side to less side. One incision was done in the greater curvature of remnant stomach at the point $60 \mathrm{~mm}$ apart from the resected line. The other incision was done on the superior edge of the duodenal transection line. After creating the $\mathrm{c}$ anastomosis lumen, the common entry incision was closed by laparoscopic linear staplers. Finally, the greater curvature of stomach and the antero-superior of duodenum were perpendicular. By using this method, the rotation duodenum and remnant stomach was not needed which can reduce the risk of poor vascular supply. In their study, there were less bile reflux, gastritis degree and residual food grade compared to DA anastomosis in 6 months after surgery.

\section{Augmented rectangle technique}

In our group, we developed a method named augmented rectangle technique (ART) anastomosis. Three automatic laparoscopic linear staplers were used to create the gastroduodenostomy and the anastomotic opening was wide and less likely to become stenosed or twisted ${ }^{[27]}$. This method was easy and time-saving. We performed 160 LDG operations using this technique from December 2013 to August 2017. There were no postoperative complications associated with the reconstruction, such as anastomotic leakage, hemorrhage or stenosis. In the ART method, the duodenum was transected form the greater curvature side to less side. Small incisions were made in duodenal stumps and the greater curvature of remnant stomach in order to insert the jaws of the linear stapler. After inserting the stapler, the lesser curvature 
Table 1. Summary of different methods applied in intracorporeal Billroth-I reconstruction

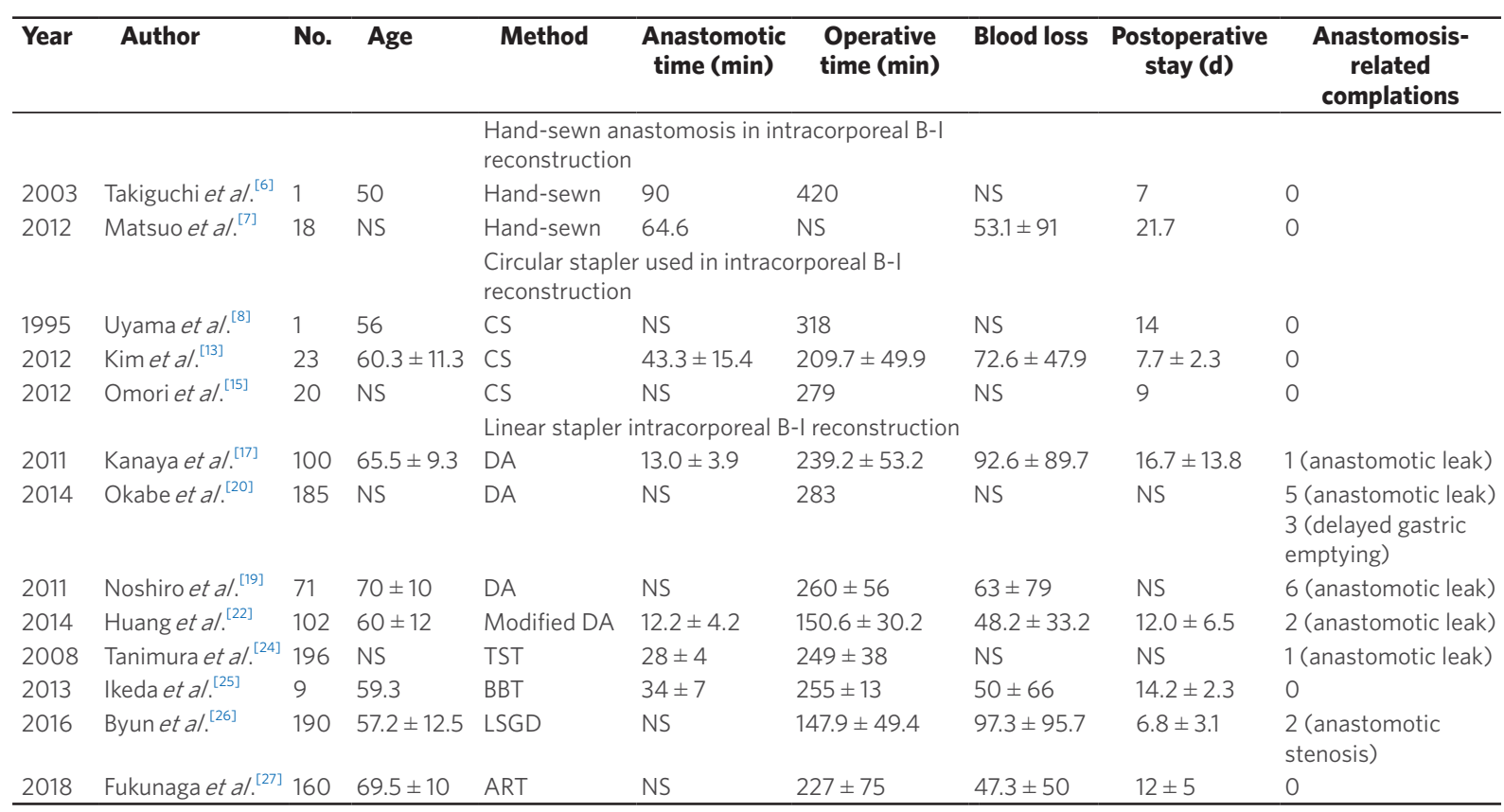

CS: Circular stapler; DA: delta-shaped anastomosis; TST: triangulating stapling technique; BBT: book-binding technique; LGSD: linearshaped gastroduodenostomy; ART: augmented rectangle technique; NS: not stated

end of the duodenal stump was rotated externally by $90^{\circ}$. After the initial suturing between the remnant stomach and the duodenum, the two sides (posterior wall and cranial wall), the posterior wall and caudal wall, form a V-shape. A $30 \mathrm{~mm}$ linear stapler was applied to close the insertion holes up to the closest side of the duodenal resection margin. After gastric and duodenal resection margins were ensured to be close together, the $60 \mathrm{~mm}$ laparoscopic linear stapler was used to transect the duodenal resection margin to create the margin. After the above steps, all the previous linear staplers were removed from duodenal resection margin.

Thanks to the elimination of the stay sutures in the anastomosis site, the risk of leakage of the intestinal contents into the peritoneal cavity can be reduced with a result of reduced incidence of peritoneal abscess $^{[28,29]}$. Removing the staple line of the duodenal stump without creating a T-shaped anastomotic region can avoid postoperative stenosis. The ART can create larger 4-sided anastomosis diameters than 3 -sided ones, without worrying about whether the width of the opening will be reduced by the final stapling.

\section{APPLICATION OF BARBED SUTURE IN INTRACORPOREAL ANASTOMOSES}

Intracorporeal suturing and knot typing in some B-I anastomosis were time-consuming and tedious and especially these procedures were the last steps to do in LDG. But various devices have been developed to simplify the placement of intracorporeal sutures, and barbed suture is one such device. Using the barber suture could reduce the number of knot typing, the suturing efficiency and reduce the cost of intracorporeal reconstruction with staplers ${ }^{[30]}$. Lee et al. ${ }^{[30]}$ used barber sutures to close entry hole in 354 patients instead of staplers with a result of minimizing the suturing time. There were no patients who needed to be converted to usual sutures or mechanical closure with staplers and only one patient presented with postoperative anastomotic bleeding.

\section{CONCLUSION}

Several reconstruction techniques are possible after TLDG [Table 1]. The best reconstruction is the one, that simplifies the technique, maintains satisfactory nutritional status and quality of life while keeping 
postoperative morbidity as low as possible. We believe that the new technologies and improved techniques will bring more benefits to patients and doctors.

\section{DECLARATIONS}

\section{Authors' contributions}

Study's conception and design: Zhang S, Fukunaga T

Writing the paper: Zhang $S$

Provided administrative, technical, and material support: Fukunaga $\mathrm{T}$

\section{Availability of data and materials}

Not applicable.

\section{Financial support and sponsorship}

The study was supported part by Japan China Sasakawa Medical Fellowship.

\section{Conflicts of interest}

All authors declared that there are no conflicts of interest.

\section{Ethical approval and consent to participate}

Not applicable.

\section{Consent for publication}

Not applicable.

\section{Copyright}

(c) The Author(s) 2019.

\section{REFERENCES}

1. Kitano S, Iso Y, Moriyama M, Sugimachi K. Laparoscopy-assisted Billroth I gastrectomy. Surg Laparosc Endosc 1994;4:146-8.

2. Bandoh T, Shiraishi N, Yamashita Y, Terachi T, Hashizume M, et al. Endoscopic surgery in Japan: the 12th national survey(2012-2013) by the Japan Society for Endoscopic Surgery. Asian J Endosc Surg 2017;10:345-53.

3. Zhang DT, Yan D, Jiang X, Song C. A modified uncut roux-en-y anastomosis in laparoscopic-assisted distal gastrectomy: balance of the cost and minimally invasion. Trans Surg 2018;3:1-5.

4. Kim MG, Kim KC, Kim BS, Kim TH, Kim HS, et al. A totally laparoscopic distal gastrectomy can be an effective way of performing laparoscopic gastrectomy in obese patients (body mass index $\geq 30$ ). World J Surg 2011;35:1327-32.

5. Piessen G, Triboulet JP, Mariette C. Reconstruction after gastrectomy: which technique is best? J Visc Surg 2010;147:e273-83.

6. Takiguchi S, Sekimoto M, Miyake Y, Fujiwara Y, Yasuda T, et al. Totally laparoscopic distal gastrectomy using the hand-sewn Billroth-I anastomotic technique: report of a case. Surg Today 2003;33:371-4.

7. Matsuo K, Shimura H, Tanaka S, Nakano M, Hashimoto T, et al. Laparoscopic distal gastrectomy with intracorporeal handsewn Billroth-I anastomosis (ICHSA). Surg Endosc 2012;26:2981-7.

8. Uyama I, Ogiwara H, Takahara T, Kato Y, Kikuchi K, et al. Laparoscopic Billroth I gastrectomy for gastric ulcer: technique and case report. Surg Laparosc Endosc 1995;5:209-13.

9. Moriya H, Shimizu S, Okano T, Yamaguchi S. Experimental study of laparoscopic gastrectomy: intracorporeal Billroth I gastroduodenostomy. Surg Laparosc Endosc 1997; 7:32-7.

10. Mayers TM, Orebaugh MG. Totally laparoscopic Billroth I gastrectomy. J Am Coll Surg 1998;186:100-3.

11. Usui S, Nagai K, Hiranuma S, Takiguchi N, Matsumoto A, et al. Laparoscopy-assisted esophagoenteral anastomosis using endoscopic purse-string suture instrument "Endo-PSI (II)" and circular stapler. Gastric Cancer 2008;11:233-7.

12. Kinoshita T, Oshiro T, Ito K, Shibasaki H, Okazumi S, et al. Intracorporeal circular-stapled esophagojejunostomy using hand-sewn purse-string suture after laparoscopic total gastrectomy. Surg Endosc 2010;24:2908-12.

13. Kim HI, Woo Y, Hyung WJ. Laparoscopic distal gastrectomy with an intracorporeal gastroduodenostomy using a circular stapler. J Am Coll Surg 2012;214:e7-13.

14. Omori T, Nakajima K, Nishida T, Uchikoshi F, Kitagawa T, et al. A simple technique for circular-stapled Billroth I reconstruction in laparoscopic gastrectomy. Surg Endosc 2005;19:734-6.

15. Omori T, Tanaka K, Tori M, Ueshima S, Akamatsu H, et al. Intracorporeal circular-stapled Billroth I anastomosis in single-incision laparoscopic distal gastrectomy. Surg Endosc 2012;26:1490-4.

16. Kanaya S, Gomi T, Momoi H, Tamaki N, Isobe H, et al. Delta-shaped anastomosis in totally laparoscopic Billroth I gastrectomy: new 
technique of intraabdominal gastroduodenostomy. J Am Coll Surg 2002;195:284-7.

17. Kanaya S, Kawamura Y, Kawada H, Iwasaki H, Gomi T, et al. The delta-shaped anastomosis in laparoscopic distal gastrectomy: analysis of the initial 100 consecutive procedures of intracorporeal gastroduodenostomy. Gastric Cancer 2011;14:365-71.

18. Kim BS, Yook JH, Choi YB, Kim KC, Kim MG, et al. Comparison of early outcomes of intracorporeal and extracorporeal gastroduodenostomy after laparoscopic distal gastrectomy for gastric cancer. J Laparoendosc Adv Surg Tech A 2011;21:387-91.

19. Noshiro H, Iwasaki H, Miyasaka Y, Kobayashi K, Masatsugu T, et al. An additional suture secures against pitfalls in delta-shaped gastroduodenostomy after laparoscopic distal gastrectomy. Gastric Cancer 2011;14:385-9.

20. Okabe H, Obama K, Tsunoda S, Tanaka E, Sakai Y. Advantage of completely laparoscopic gastrectomy with linear stapled reconstruction: a long-term follow-up study. Ann Surg 2014;259:109-16.

21. Lee Y, Tan CH, Park DJ. Current status of intracorporeal gastroduodenostomy and modified delta-shape anastomosis after distal gastrectomy for gastric cancer. J Vis Surg 2016;2:158.

22. Huang C, Lin M, Chen Q, Lin J, Zheng C, et al. A modified delta-shaped gastroduodenostomy in totally laparoscopic distal gastrectomy for gastric cancer: a safe and feasible technique. PLoS One 2014;9:e102736.

23. Huang CM, Lin M, Lin JX, Zheng CH, Li P, et al. Comparision of modified and conventional delta-shaped gastroduodenostomy in totally laparoscopic surgery. World J Gastroenterol 2014;20:10478-85.

24. Tanimura S, Higashino M, Fukunaga Y, Takemura M, Nishikawa T, et al. Intracorporeal Billroth 1 reconstruction by triangulating stapling technique after laparoscopic distal gastrectomy for gastric cancer. Surg Laparosc Endosc Percutan Tech 2008;18:54-8.

25. Ikeda T, Kawano H, Hisamatsu Y, Ando K, Saeki H, et al. Progression from laparoscopic-assisted to totally laparoscopic distal gastrectomy: comparison of circular stapler (i-DST) and linear stapler (BBT) for intracorporeal anastomosis. Surg Endosc 2013;27:325-32.

26. Byun C, Cui LH, Son SY, Hur H, Cho YK, et al. Linear-shaped gastroduodenostomy (LSGD): safe and feasible technique of intracorporeal Billroth I anastomosis. Surg Endosc 2016;30:4505-14.

27. Fukunaga T, Ishibashi Y, Oka S, Kanda S, Yube Y, et al. Augmented rectangle technique for Billroth I anastomosis in totally laparoscopic distal gastrectomy for gastric cancer. Surg Endosc 2018; doi: 10.1007/s00464-018-6266-1.

28. Jeong O, Jung MR, Park YK, Ryu SY. Safety and feasibility during the initial learning process of intracorporeal Billroth I (delta-shaped) anastomosis for laparoscopic distal gastrectomy. Surg Endosc 2015;29:1522-9.

29. Lin M, Zheng CH, Huang CM, Li P, Xie JW, et al. Totally laparoscopic versus laparoscopy-assisted Billroth-I anastomosis for gastric cancer: a case-control and case-matched study. Surg Endosc 2016;30:5245-54.

30. Lee SW, Kawai M, Tashiro K, Bouras G, Kawashima S, et al. Laparoscopic distal gastrectomy with D2 lymphadenectomy followed by intracorporeal gastroduodenostomy for advanced gastric cancer: technical guide and tips. Transl Gastroenterol Hepatol 2017;2:84. 